\title{
RACSAM
}

Rev. R. Acad. Cien. Serie A. Mat.

VOL. 103 (1), 2009, pp. 91-91

Análisis Matemático / Mathematical Analysis

\section{Addendum to the paper The Projective Tensor Product II: The Radon-Nikodym Property}

\author{
Joe Diestel, Jan Fourie, and Johan Swart
}

The paper The Projective Tensor Product II: The Radon-Nikodym Property, by Joe Diestel, Jan Fourie, and Johan Swart, was published in Rev. R. Acad. Cien. Serie A. Mat. Vol. 100 (1-2), 2006, pp. 75-100, submitted by Vicente Montesinos. The authors have sent the following addendum to the page 96 :

Replace lines 19 to 23 in page 96 , i.e., from:

to

“( $B_{Y^{* *}}$, weak $\left.k^{*}\right)$. But it's easy..."

"is $v$-measurable and

$$
G_{2}=H_{2} \cdot \chi_{\left(B_{X * *}, w_{e a k^{*}}\right)} \times B_{Y} "
$$

by

“ $\left(B_{Y^{* *}}\right.$, weak $\left.k^{*}\right)$. To see that $\left(B_{X^{* *}}\right.$, weak $\left.k^{*}\right) \times B_{Y}$ is $v$-measurable, we call on Choquet's theory of capacities and $\mathcal{K}$-analytic sets. More specifically, since $B_{Y}$ is Polish it is a $\mathcal{K}_{\sigma \delta}$ set; it follows that $\left(B_{X^{* *}}\right.$, weak $\left.k^{*}\right) \times B_{Y}$ is a $\mathcal{K}_{\sigma \delta}$ subset of $K$, its Cech-Stone compactification. As such it is universally " $f$-capacitable", where $f$ ranges over all the regular capacities defined on $K$; in particular, it is universally measurable with respect to the regular Borel measures on $K$ [cf. G. Choquet, Lectures on Analysis, Vol. I, W. A. Benjamin, Inc., 1969, especially pp. 141-156]. Moreover

$$
G_{2}=H_{2} \cdot \chi_{\left(B_{X * *}, w_{e a k}^{*}\right)} \times B_{Y} "
$$

\footnotetext{
*This addendum was received by Professor Vicente Montesinos on 20th March 2009 and its publication was accepted in 1th April
} 2009. 